\title{
How Hyaluronan Could Reduce Inflammation During Arthritis? What's New?
}

\section{Giuseppe Maurizio Campo*}

Department of Biomedical Sciences and Morphological and Functional Images, School of Medicine, University of Messina, Policlinico Universitario, 98125 - Messina, Italy

Chronic inflammation, characterized by elevated circulating levels of inflammatory markers, appears to play a critical role in the pathogenesis of arthritis and its associated complications, particularly the autoimmune process that wreaks havoc on the joints can also affect the eyes, lungs, skin, heart and blood vessels, and other organs [1].

Although articular injection of Hyaluronan (HA) is frequently used in the treatment of arthritic conditions, the precise mechanism by which HA suppresses degradation of cartilage or intra-articular inflammation is still under vigorous investigation. A disease-modifying effect of HA has been suggested by some in vitro studies; however, little clinical evidence has been provided thus far. A better understanding of the wide network of signalling pathways of anti-inflammatory effects by HA will encourage novel anti-degradation strategies for both inflammatory and degenerative arthritides $[2,3]$.

The cluster determinant $44(\mathrm{CD} 44)$ is a transmembrane glycoprotein widely distributed on $\mathrm{T}$ lymphocytes, granulocytes, monocytes, fibroblasts, keratinocytes, chondrocytes and epithelial cells. Regulation of the CD44 interaction with its ligands depends highly on Protein Kinase C (PKC) which modulates the phosphorylation state of CD44 and the various anchoring proteins. Therefore, PKC has the potential to modulate both the affinity of CD44 for its ligands as well as the localization of the receptor over the cell surface [4]. It has also been reported that the activation of the CD44 receptor by HA produced PKC activation, thus establishing a concatenated mechanism between the CD44 receptor and PKC [5]. In addition, these processes are to a large extent mediated by the increased expression of pro-inflammatory cytokines and other detrimental molecules such as nitric oxide (NO) and matrix metalloproteinases (MMPs), which are in turn controlled by the activation of key transcriptional nuclear factor kB (NF-kB). NF-kB, in turn, promotes the transcription of several genes with production of different molecules, including cytokines and other inflammation products that chronically damage tissue and organs [5].

Toll-Like Receptors (TLRs) are the family of trans-membrane receptors involved in innate immunity and pathogen recognition. The role of TLRs in both innate and adaptive immunity suggests that genetic variations within genes encoding these receptors have an important influence on the pathogenesis of inflammatory diseases [6]. One of the central receptors of the innate immune response is the Toll-like receptor 4 (TLR4) which is expressed on a variety of cells and tissues. TLR4 plays an important role in the recognition of microbial components, particularly Lipopolysaccharides (LPS) [7]. It also interacts with endogenous ligands that are present during inflammation, e.g. oxidized LDL, heat shock proteins 60 and 70, fibrinogen, fibronectin and HA [8]. TLR4 stimulation activates the innate immune response via the NF-kB pathway and subsequent pro-inflammatory reactions [7].

Regulation of tissue injury and repair is a carefully orchestrated host response which is designed to eradicate the offending agent and restore tissue integrity. The mechanisms that regulate the response to tissue damage are not fully understood. Abnormalities in the repair response are associated with a variety of chronic disease states characterized by inflammation, followed later by Excessive Extracellular Matrix (ECM) deposition and finally by tissue fibrosis [9]. In concert with initiating the inflammatory response, biochemical signals are also generated in order to minimize the extent of cell damage. The ultimate outcome depends on the balance between containment of injury, maintenance of structural cell integrity, and activation of repair mechanisms [9]. The dynamic turnover of ECM results in the net deposition of matrix in the interstitium of the damaged tissue. An additional consequence of the degradation of ECM components is the generation of fragments from larger precursor molecules. A major component of the ECM that undergoes dynamic regulation during tissue injury and inflammation is the non-sulphated Glycosaminoglycan (GAG) HA [10]. HA is a linear polymer composed of repeating disaccharides $D$-glucuronic acid $-N$-acetyl $D$-glucosamine. HA may exist both as a soluble polymer as well as in non-covalently linked complexes with proteins called HAbinding proteins or hyaladherins. HA exists as a high-molecularweight polymer $\left(10^{6} \mathrm{D}\right)$ under physiological conditions [10]. However, under inflammatory conditions HA has been shown to be more polydisperse in size, with a preponderance of lower molecular weight forms, especially after tissue injury [11]. Several findings have shown that HA functions are determined by the size of the molecule, in addition to the structure and its interaction with HA binding proteins. High molecular weight HA is suggested to play a structural role and to promote tissue integrity, while low molecular weight HA may be a signal of tissue injury [12].

Cell interaction with the surrounding extracellular matrix is fundamental in many physiological and pathological mechanisms. Proteoglycans (PGs) may influence cell behaviour through binding events mediated by their GAG chains. The binding affinity of the interaction depends on the ability of the oligosaccharide sequence to provide an optimal charge and surface with the protein [13]. It has been demonstrated that the interaction of HA degradation products with CD44 provides signals to initiate inflammation [14]. Other data support the idea that a balance between low molecular weight HA and high molecular weight $\mathrm{HA}$ amounts may control the activation of inflammation. In particular, high molecular weight HA is able to maintain homeostasis and potentially down-regulate inflammation [15]. We previously reported that high molecular weight HA was able to limit inflammation CD44-activated stimulated by Phorbol-

*Corresponding author: Giuseppe M Campo, $\mathrm{PhD}$, Department of Biomedical Sciences and Morphological and Functional Images, School of Medicine, University of Messina, Policlinico Universitario, Torre Biologica, $5^{\circ}$ piano, Via C. Valeria - 98125 Messina, Italy, Tel: +39 090221 3334; Fax: +39 090221 3898; E-mail: gcampo@unime.it

Received July 25, 2012; Accepted July 26, 2012; Published July 28, 2012

Citation: Campo GM (2012) How Hyaluronan Could Reduce Inflammation During Arthritis? What's New? J Arthritis 1:e103. doi:10.4172/2167-7921.1000e103

Copyright: (c) 2012 Campo GM. This is an open-access article distributed unde the terms of the Creative Commons Attribution License, which permits unrestricted use, distribution, and reproduction in any medium, provided the original author and source are credited. 
12-Myristate-13-Acetate (PMA) in mouse chondrocytes. By using a specific CD44 blocking antibody it was demonstrated that CD44 receptor was not the HA target, while the target for HA action was activated PKC [16]. We also reported that high molecular weight HA was able to reduce TLR4- activated inflammation, stimulated by LPS in mouse chondrocytes. In this case the use of specific blocking antibody demonstrated that TLR4 was the target of high molecular weight HA [17]. It is the number of active charged HA sites (carboxylic groups) that are responsible for HA action. In fact, high molecular weight HA containing many charged groups may completely envelop protein structures such as PKC or TLR-4, thereby preventing their activation. The mechanism could be similar to the well known lock and key model that applies to all well-known effector-receptors interactions where the effector is structurally and chemically highly specific so that a similar molecule with equally distributed charged groups may block the receptor. In this case, the larger size of HA molecule was able to completely mask the active site of PKC or TLR-4. Therefore, the inhibition of PKC or TLR4 produced a reduction in NF-kB activation that in turn limited the transcription of the detrimental inflammatory intermediates with a consequent reduction in cell and tissue injury.

Adenosine $(\mathrm{ADO})$ is an endogenous mediator that typically serves as a cytoprotective modulator in response to stress [18]. It is a nucleoside formed by the enzymatic breakdown of ATP, especially during tissue injury [18]. It has been reported that tissue damage and inflammation are accompanied by the accumulation of extracellular adenosine due to its release from non immune and immune cells [19]. The rapid release of $\mathrm{ADO}$ in response to tissue-disturbing stimuli has a dual role in modulating homeostasis. First, extracellular ADO represents a pre-eminent alarm molecule that reports tissue injury in an autocrine and paracrine manner to surrounding tissue. Second, extracellular ADO generates a range of tissue responses that can be generally viewed as organ protective thereby mediating homeostasis. ADO interacts with four different $G$-protein-coupled receptors: $A_{1}$, $A_{2 A}, A_{2 B}$, and $A_{3}$. The selective engagement of the $A_{2 A}$ receptor by ADO activates stimulatory G-proteins that activate adenylate cyclase, leading to increased cAMP production [19]. Although the exact signal transduction pathways used by $\mathrm{ADO}$ have not yet been fully elucidated, elevated levels of cAMP have been associated with activation of protein kinase A (PKA) and inhibition of NF-kB [20]. The anti-inflammatory effects can be achieved by increasing intracellular or extracellular ADO levels through the mechanism of either enhanced production or inhibition of ADO catabolism. The ADO receptor system has evolved as both a rapid sensor of tissue injury and the major 'first-aid' machinery of tissues and organs. ADO receptor activation thus preserves tissue function and prevents further tissue injury following an acute injurious insult, such as reperfusion injury, actions in which the immune system has a paramount role. This primordial protective function of the ADO receptor system following acute insults can, however, be overshadowed by its reduced ability to protect against chronic insults. The majority of works has been focusing on inhibition of ADO catabolism or direct activation of ADO receptors. ADO has been shown to limit systemic inflammatory responses through the receptor-mediated regulation of a great variety of cell types. One of the best documented antiinflammatory functions of $\mathrm{ADO}$ is its ability to regulate cytokine synthesis. The production of pro-inflammatory cytokines, including TNF- $\alpha$, interleukin-8 (IL-8), macrophage inflammatory protein-1alpha (MIP-1a, interleukin-2 (IL-2) and IL-6, is suppressed by exposure to $\mathrm{ADO}$ or $\mathrm{ADO}$ analogues [21]. Conversely, exposure to $\mathrm{ADO}$ increases the synthesis of interleukin-10 (IL-10), a potent regulator of macrophage function. Hence, strategies to stimulate $\mathrm{A}_{2 \mathrm{~A}} \mathrm{R}$ would seem to be an interesting approach for reducing the inflammatory response.

The innovation of this scientific approach comes from the fact that these pathways in arthritis are unexplored, although CD44, PKC and TLRs involvement has been well-documented previously. In addition, since HA is an endogenous molecule, by using high molecular weight $\mathrm{HA}$ as modulator of these structures, its action could modulate these structures in a physiological way more effectively than other exogenous compounds. The expected findings of this new approach could have several scientific implications, starting from the effects on the course of arthritic pathologies, such as rheumatoid arthritis, osteoarthritis and their complications in vivo that may result from blocking CD44, PKCalpha, PKCbeta1, PKCbeta2 and PKCdelta and TLRs structures with specific antibodies. Furthermore, the expression of inflammatory mediators after inhibition/modulation of CD44, PKCalpha, PKCbeta1, PKCbeta2 and PKCdelta and TLRs with HA could provide fundamental information for the future development of specific drugs, with a structure similar to HA, designed to curb the complications of arthritis. Important information could also come out by analysing the expression of other TLRs in addition to TLR4, such as TLR1, TLR2 and TLR3 which all seem to be implicated in inflammatory processes. Another important finding of the present study is that triggering of the adenosine A2A receptor may represent a rational therapeutic strategy to achieve the goal of a complete inhibition of the effect by several early and late inflammatory cytokines involved in the pathogenesis of rheumatoid arthritis [22,23]. As well as for HA, the anti-inflammatory mechanism exerted by adenosine needs to be better elucidated in order to focus pharmacological strategies in this direction. The intricate and complex inflammation mechanism seems to be due to a delicate equilibrium in which pro-inflammatory pathways, induced by agents such as HA oligosaccharides derived from native HA degradation during inflammation, coexist with antiinflammatory pathways induced by molecules such as the extracellular endogenous adenosine released during inflammatory diseases. The modulation of these pathways could be a useful tool for modulating inflammation. It has been suggested that the use of adenosine agonists may reduce inflammation in different types of experimental models, and that inhibition of the action of low molecular mass HA leads to an inhibition of the inflammatory mechanism. Therefore, we believe that by acting on degraded HA pathways with the appropriate tools, together with the use of other opportune and specific tools on adenosine pathways, would enable the intricate mechanism of inflammation to be clarified and better understood. At the same time, this would allow anti-inflammatory strategies to be developed to tackle inflammation more effectively than currently available therapeutic drugs.

\section{References}

1. Lee DM, Weinblatt ME (2001) Rheumatoid arthritis. Lancet 358: 903-911.

2. Campo GM, Avenoso A, D'Ascola A, Scuruchi M, Prestipino V, et al. (2012) The inhibition of hyaluronan degradation reduced pro-inflammatory cytokines in mouse synovial fibroblasts subjected to collagen-induced arthritis. J Cell Biochem 113: 1852-1867.

3. Campo GM, Avenoso A, D'Ascola A, Prestipino V, Scuruchi M, et al. (2012) Inhibition of hyaluronan synthesis reduced inflammatory response in mouse synovial fibroblasts subjected to collagen-induced arthritis. Arch Biochem Biophys 518: 42-52.

4. Newton AC (2003) Regulation of the ABC kinases by phosphorylation: protein kinase $\mathrm{C}$ as a paradigm. Biochem J 370: 361-371.

5. Naor D, Nedvetzki S, Walmsley M, Yayon A, Turley EA (2007) CD44 involvement in autoimmune inflammations: the lesson to be learned from CD44-targeting by antibody or from knockout mice. Ann NY Acad Sci 1110: 233-247. 
Citation: Campo GM (2012) How Hyaluronan Could Reduce Inflammation During Arthritis? What's New? J Arthritis 1:e103. doi:10.4172/21677921.1000 e103

Page 3 of 3

6. Hughes AL, Piontkivska H (2008) Functional diversification of the toll-like receptor gene family. Immunogenetics 60: 249-256.

7. Riddel JR, Wang XY, Minderman H, Gollnick SO (2010) Peroxiredoxin 1 stimulates secretion of proinflammatory cytokines by binding to TLR4. J Immunol 184:1022-1030.

8. Akira S, Takeda K, Kaisho T (2001) Toll-like receptors: Critical proteins linking innate and acquired immunity. Nat Immunol 2: 675-680.

9. Kaczorowaski DJ, Mollen KP, Edmonds R, Billiar TR (2008) Early events in the recognition of danger signals after tissue injury. J Leukoc Biol 83: 546-552.

10. Fraser JR, Laurent TC, Laurent UB (1997) Hyaluronan: its nature, distribution, function and turnover. J Intern Med 242: 27-33.

11. Noble PW (2002) Hyaluronan and its catabolic products in tissue injury and repair. Matrix Biol 21: 25-29.

12. Jiang D, Liang J, Noble PW (2007) Hyaluronan in tissue injury and repair. Annu Rev Cell Dev Biol 23: 435-461.

13. Yates EA, Terry CJ, Rees C, Rudd TR, Duchesne L, et al. (2006) ProteinGAG interactions: new surface-based techniques, spectroscopies and nanotechnology probes. Biochem Soc Trans 34: 427-430.

14. Heldin P, Karousou E, Bernert B, Porsch H, Nishitsuka K, et al. (2008) Importance of hyaluronan-CD44 interactions in inflammation and tumorigenesis. Connect Tissue Res 49: 215-218.

15. Steeber DA, Venturi GM, Tedder TF (2005) A new twist to the leukocyte adhesion cascade: intimate cooperation is key. Trends Immunol 26: 9-12.
16. Campo GM, Avenoso A, Campo S, D'Ascola A, Traina P, et al. (2009) Differential effect of molecular size HA in mouse chondrocytes stimulated with PMA. Biochim Biophys Acta 1790: 1353-1367.

17. Campo GM, Avenoso A, Campo S, D'Ascola A, Nastasi G, et al. (2010) Molecular size hyaluronan differently modulates toll-like receptor-4 in LPSinduced inflammation in mouse chondrocytes. Biochimie 92: 204-215.

18. Jacobson KA (2009) Introduction to adenosine receptors as therapeutic targets. Handb Exp Pharmacol 193: 1-24.

19. Sitkovsky MV (2003) Use of the A(2A) adenosine receptor as a physiological immunosuppressor and to engineer inflammation in vivo. Biochem Pharmacol 65: 493-501.

20. Clementina M, Giuseppe S (2010) A2A receptor ligands: past, present and future trends. Curr Top Med Chem 10: 902-922.

21. Varani K, Padovan M, Vincenzi F, Targa M, Trotta F, et al. (2011) A2A and A3 adenosine receptor expression in rheumatoid arthritis: upregulation, inverse correlation with disease activity score and suppression of inflammatory cytokine and metalloproteinase release. Arthritis Res Ther 13: R197.

22. Campo GM, Avenoso A, D'Ascola A, Scuruchi M, Prestipino V, et al. (2012) Adenosine $\mathrm{A} 2 \mathrm{~A}$ receptor activation and hyaluronan fragment inhibition reduce inflammation in mouse articular chondrocytes stimulated with interleukin-1 $\beta$. FEBS J 279: 2120-2133.

23. Campo GM, Avenoso A, D'Ascola A, Prestipino V, Scuruchi M, et al. (2012) The stimulation of adenosine $2 \mathrm{~A}$ receptor reduces inflammatory response in mouse articular chondrocytes treated with hyaluronan oligosaccharides. Matrix Biol. 